\title{
ON LINEAR SPACES WHICH MAY BE RENDERED COMPLETE NORMED METRIC SPACES
}

\section{CASPER GOFFMAN}

In this paper, we obtain a characterization of linear spaces which may be normed so as to become complete, linear, normed metric spaces. In this connection, K. Kunugui ${ }^{1}$ and M. Fréchet ${ }^{2}$ have shown that every metric space $S$ is isometric with a subset of a complete, linear, normed metric space. It follows from our result that if the cardinal number of $S$ is the limit of a denumerable sequence of cardinals, then there is no complete, linear, normed metric space isometric with $S$. Results on topological spaces which may be rendered linear, normed metric spaces and complete, linear, normed metric spaces have been given by $\mathrm{A}$. Kolmogoroff ${ }^{3}$ and B. Z. Vulich.

It will be assumed that the reader is familiar with certain elementary portions of the theory of linear and metric spaces, and with transfinite cardinal and ordinal numbers. ${ }^{5}$ Using the generalized continuum hypothesis and normal order theorem, we prove the following:

Theorem. A necessary and sufficient condition that a linear space may be made a complete, linear, normed metric space by a suitable definition of norm is that the cardinal number of its Hamel basis should not be the limit of any denumerable sequence of cardinals which precede it.

A Hamel basis of a linear space $S$ is a subset $T$ of $S$ such that every element of $S$ is a linear combination, with real coefficients, of a finite number of elements of $T$, and there is no proper subset of $T$ with this property. The following properties of a Hamel basis will be used in demonstrating the theorem, and are given without proof:

(a) A linear space $S$ has a Hamel basis. ${ }^{6}$

Received by the editors October 25, 1942.

${ }^{1} \mathrm{~K}$. Kunugui, Applications des espaces à une infinitê de dimensions à la thêorie des ensembles, Proc. Imp. Acad. Tokyo vol. 11 no. 9 pp. 351-353.

2 M. Fréchet, Sur les espaces distanciés. Memorial volume dedicated to D. A. Grave. Moscow, 1940, pp. 265-267.

${ }^{3}$ A. Kolmogoroff, Zur Normierbarkeit eines allgemeinen topologischen Raumes, Studia Mathematica, vol. 5 (1934) pp. 29-33.

${ }^{4}$ B. Z. Vulich, Linear spaces with given convergence, Leningrad State University Annals, Mathematics Series vol. 10 (1940) pp. 40-63.

5 For the elements of transfinite number theory, see F. Hausdorff, Mengenlehre, Berlin, 1927; for the theory of linear spaces, see S. Banach, Théorie des opérations linéaires, Warsaw, 1932.

6 Proofs of (a) and (b) have been given by H. Löwig, Über die Dimension linearer Räume, Studia Mathematica, vol. 5 (1934) pp. 18-24. 
(b) $S$ may have many distinct bases, but these must have the same cardinal number.

(c) If the basis $T$ of $S$ is nondenumerable, then $s$ and $t$, the respective cardinals of $S$ and $T$, are identical.

(d) The representation of every element $x$ of $S$ as a linear combination, with nonzero coefficients, of a finite number of elements of a given basis $T$ of $S$ is unique.

1. Sufficiency. We note that a linear, normed metric space with a finite Hamel basis, of cardinal $n$, is homeomorphic with euclidean $n$-space; hence, is complete. To prove the sufficiency part of the theorem we need, therefore, consider only the infinite case; that is, we must show that a linear space whose basis is of infinite cardinal not the limit of any denumerable sequence of cardinals which precede it may be rendered complete by a suitable definition of norm. Such cardinals are either non-limiting, infinite cardinals, or limiting cardinals which are not the limit of any denumerable sequence of cardinals. First, let $\alpha$ be a non-limiting, infinite cardinal, and $\beta$ its immediate predecessor in the normal order of cardinals. By the generalized continuum hypothesis, $2^{\beta}=\alpha$. Let $S$ be the linear space of all bounded, real functions defined on a given set of $\operatorname{cardinal} \beta$, the sum of two functions and the product of a function by a real number being defined in the customary way. $S$ is of cardinal $c^{\beta}=2^{\beta}=\alpha$. With $\nu(f)=1$.u.b. $|f(x)|$, the least upper bound of $|f(x)|$, as norm, $S$ is evidently a complete, linear, normed metric space. For $\alpha>c$, as previously noted, every basis of $S$ has the same cardinal $\alpha$ as $S$. For $\alpha=c, S$ certainly has no finite basis and, since complete, has no denumerable basis, as will be shown in the proof of the necessity part of the theorem. Hence, by the continuum hypothesis, if $S$ is of cardinal $c$, its basis is also of cardinal $c$. We have thus defined, for every non-limiting, infinite cardinal $\alpha$, a complete, linear, normed metric space with basis of cardinal $\alpha$. Any linear space $S^{\prime}$ with basis of cardinal $\alpha$ is isomorphic with $S$. For, if $T$ and $T^{\prime}$ are respective bases of $S$ and $S^{\prime}$, a biunique correspondence exists between them. It then follows from the uniqueness of the representation of the elements of a space as a linear combination of basal elements, that $S$ and $S^{\prime}$ are isomorphic linear spaces. Hence, by letting the norm of every element of $S^{\prime}$ be identical with that of its mate in $S$, the linear space $S^{\prime}$ is made complete. Now, let $\alpha$ be a limiting, infinite cardinal which is not the limit of any denumerable sequence of cardinals which precede it. For every cardinal $\beta<\alpha$, let $T_{\beta}$ be a set of cardinal $\beta$ such that, for every $\gamma<\beta$, $T_{\gamma}$ is a subset of $T_{\beta}$. Such a class of sets may be obtained, for ex- 
ample, by normally ordering a set of cardinal $\alpha$, and letting $T_{\beta}$ be the proper initial segment of the order which introduces the cardinal $\beta$. Let $T=\sum_{\beta<\alpha} T_{\beta}$ and let $S_{\beta}$ be the set of bounded, real functions defined over $T$, arbitrarily on $T_{\beta}$ and identically zero on $T-T_{\beta}$. The cardinal number of $S_{\beta}$ is the immediate successor of $\beta$, by the generalized continuum hypothesis, and the cardinal number of $S=\sum_{\beta<\alpha} S_{\beta}$ is $\alpha$. As in the preceding case, $S$ may be rendered a linear, normed metric space by defining the sum of two functions of $S$ and the product of a function of $S$ by a real number in the manner customar for functions, and by letting $\nu(f)=1$.u.b. $|f(x)|$, for every $f(x)$ in $S$. The space $S$ is a complete metric space. For, let $\left\{f_{n}(x)\right\}$ be any convergent sequence of elements of $S$. Then $\left\{f_{n}(x)\right\}$ is a uniformly convergent sequence of functions. Let $\beta_{n}$ be the smallest cardinal such that $f_{n}(x)$ is identically zero on the set $T-T_{\beta_{n}}$. Then $\beta_{n}<\alpha$ and, since $\alpha$ is not the limit of any denumerable sequence of cardinals which precede it, there exists a cardinal $\beta<\alpha$ such that $\beta_{n}<\beta$, for all $n$. The functions of the sequence $\left\{f_{n}(x)\right\}$ are thus identically zero on $T-T_{\beta}$ and, since the sequence is convergent, its limit, $f(x)$, is also identically zero on $T-T_{\beta}$. Moreover, $f(x)$ is bounded, for it is the limit of a uniformly convergent sequence of bounded functions. Hence, $f(x)$ is in $S_{\beta}$ and thus in $S$. The linear, normed metric space $S$ is therefore complete. It then follows from the isomorphism of linear spaces whose bases are of the same cardinal, that every linear space with basis of cardinal $\alpha$ may be normed so as to be complete.

2. Necessity. We show that a linear, normed metric space is not a complete metric space if the cardinal number of its basis is the limit of a denumerable sequence of cardinals which precede it. We prove the following:

LEMMA. If $T$ is a nondense, linear subspace of a linear, normed metric space $S$, then $T$ is nowhere dense in $S$.

Proof. Let $\sigma$ be a sphere of elements of $S$. Then $c \sigma$, the set obtained by multiplying the elements of $\sigma$ by a real number $c \neq 0$, and $x+\sigma$, the set obtained by adding to the elements of $\sigma$ a given element $x$ of $S$, are also spheres in $S$. Since $T$ is nondense in $S$, there is a sphere $\sigma$ in $S$, containing no point of $T$. Let $\left\{c_{n}\right\}$ be a sequence of reals converging to zero. Then, for every $x$ in $T$, the sequence $\left\{x+c_{n} \sigma\right\}$ of spheres converges to $x$. But these spheres contain no points of $T$. Hence, $T$ is nowhere dense in $S$.

Suppose $S$ is a linear, normed metric space with a denumerable basis, whose elements are $\xi_{1}, \xi_{2}, \ldots, \xi_{n}, \cdots$. Let $S_{n}$ be the linear 
subspace of $S$ with basis $\xi_{1}, \xi_{2}, \cdots, \xi_{n}$. Then $S=\sum_{1}^{\infty} S_{n}$. Since $S_{n}$ has a finite basis, it is a complete metric space; hence, closed in $S$. But a closed subset of a metric space is nondense in the space. Therefore, by the lemma, $S_{n}$ is nowhere dense in $S$, and $S$, as the limit of $\boldsymbol{\aleph}_{0}$ nowhere dense sets, is of the first category, and therefore not complete.

Finally, we construct, for every nondenumerable cardinal $\alpha$ which is the limit of a denumerable sequence of cardinals, a linear space of cardinal $\alpha$, and hence, with basis of cardinal $\alpha$, which cannot be normed so as to be rendered complete. Since, as noted above, two linear spaces are isomorphic if their bases are of the same cardinal, it follows that every linear space of cardinal $\alpha$ has the desired property. Now, let $\left\{\alpha_{n}\right\}$ be the sequence of consecutive, increasing cardinals which converges to $\alpha$, and let $T_{n}$ be a set of cardinal $\alpha_{n}$ such that, for every $n, T_{n}$ is a subset of $T_{n+1}$. Let $T=\sum_{1}^{\infty} T_{n}$. Let $S_{n}$ be the set of bounded, real functions defined over $T$, arbitrarily on $T_{n}$ and zero elsewhere, and let $S=\sum_{1}^{\infty} S_{n}$. The cardinal number of $S_{n}$ is $2^{\alpha_{n}}=\alpha_{n+1}$, by the generalized continuum hypothesis, and the cardinal number of $S$ is $\alpha=\sum_{1}^{\infty} \alpha_{n+1} . S$ is rendered a linear space, if the sum of two functions and the product of a function by a real number are defined in the usual way. Suppose $S$ is further rendered normed and metric by any given choice of norm for the elements of $S$. $S_{n}$ is obviously a linear, normed metric proper subspace of $S$. Moreover, $S_{n}$ is a nondense subset of $S$. For there are only $\alpha_{n+1}^{\aleph_{0}}=\alpha_{n+1}$ sequences composed of elements of $S_{n}$ and, since $S$ is of cardinal $\alpha>\alpha_{n+1}$, there exists an element of $S$ not a limit point of $S_{n}$. By the lemma, $S_{n}$ is nowhere dense in $S$ and $S=\sum_{1}^{\infty} S_{n}$ is of the first category. Hence $S$ is not complete.

As a consequence of the necessity part of this theorem, it immediately follows that a metric space whose cardinal number is the limit of no denumerable sequence of cardinals is homeomorphic, hence isometric, with no complete, linear, normed metric space.

Pittsburgh, Pa. 